\title{
Corela
}

Cognition, représentation, langage

4-1 | 2006

Vol. $4, \mathrm{n}^{\circ} 1$

\section{La nature du procès dans les énoncés en BE + -ING}

\section{Laurence Vincent-Durroux}

\section{OpenEdition}

\section{Journals}

Édition électronique

URL : http://journals.openedition.org/corela/418

DOI : $10.4000 /$ corela.418

ISSN : 1638-573X

\section{Éditeur}

Cercle linguistique du Centre et de l'Ouest - CerLICO

\section{Référence électronique}

Laurence Vincent-Durroux, «La nature du procès dans les énoncés en BE + -ING », Corela [En ligne] 4-1 | 2006, mis en ligne le 28 juin 2006, consulté le 02 mai 2019. URL : http://journals.openedition.org/ corela/418; DOI : 10.4000/corela.418

Ce document a été généré automatiquement le 2 mai 2019.

\section{(c) (i) (2)(2)}

Corela - cognition, représentation, langage est mis à disposition selon les termes de la licence Creative Commons Attribution - Pas d'Utilisation Commerciale - Partage dans les Mêmes Conditions 4.0 International. 


\title{
La nature du procès dans les énoncés en $B E+-I N G$
}

\author{
Laurence Vincent-Durroux
}

1 Il est fréquent de considérer que $\mathrm{BE}+$ - ING connait des restrictions à son emploi ; certains verbes sont ainsi répertoriés comme étant difficilement compatibles voire non compatibles avec BE +-ING. La nature du procès est souvent mise en avant et les procès de type état figurent parmi les principales "exceptions" à l'emploi de BE +-ING. Toutefois, il n'est guère satisfaisant de rendre compte des phénomènes en les présentant comme des "exceptions".

2 C'est pourquoi nous proposons ici d'interroger la distinction état / processus et de formuler une hypothèse sur la détermination dont $\mathrm{BE}+$ - ING est le marqueur, afin de rendre compte de la moindre fréquence de certains procès avec $\mathrm{BE}+-\mathrm{ING}$, sans que ceuxci soient à considérer comme des "exceptions". Nos analyses s'appuient majoritairement sur les théories de l'énonciation'1.

\section{Remarques liminaires et hypothèse}

\subsection{Sortir d'un "raisonnement circulaire"}

3 Le fait d'exclure certains procès de l'emploi de BE + -ING pourrait relever d'un " raisonnement circulaire" clairement explicité par Bouscaren et Deschamps (1991, pp. 9-10) :

La valeur des procès dans les énoncés dépend de deux paramètres distincts :

- d'un côté une typologie des notions qui reflète les propriétés physico-culturelles ("aktionsart"),

- de l'autre les opérations énonciatives qui permettent de construire des énoncés ancrés dans une situation. Tous les opérateurs utilisés (temps, aspects, modalités, complémentation, détermination) vont contribuer à l'interprétation de l'énoncé.

- La confusion entre les deux paramètres est souvent à la base de raisonnements circulaires ${ }^{2}$ où l'on postule par exemple qu'un verbe statif en anglais est un verbe qui ne s'utilise pas à la 
forme en be + ing, et que par ailleurs la forme en be + ing ne s'utilise pas avec les verbes statifs. On ne peut à la fois définir un type de procès ${ }^{3}$ en référence à une structure syntaxique et caractériser la structure syntaxique comme s'appliquant à ce type de procès. notions" (Aktionsart) et les "opérations énonciatives" portant sur l'aspect. Si nous retenons les définitions de Palmer (citées par Furuli, 1997) : "Aktionsart describes a class of action. Aspect describes a way of viewing an action", et de Furuli (1997): "the way [an] action is perceived by a language user" pour l'Aktionsart, et: "the way that language user decides to portray that action" pour l'aspect, nous relevons que seul l'aspect se situe strictement au plan de la représentation linguistique. Ce n'est pas le cas de l'Aktionsart, puisqu'il est impossible de savoir avec certitude comment l'action est perçue, ainsi que le remarque fort justement Furuli.

5 Or, à quels niveaux la catégorisation en procès de type état et procès de type processus est-elle pertinente : au niveau de la perception (Aktionsart), de la représentation (aspect) ou encore de la représentation de la représentation (niveau métalinguistique) ? Se pose aussi la question de savoir si la détermination dont BE + -ING est le marqueur affecte la distinction entre état et processus.

\subsection{Quelques approches relevées dans la littérature}

\subsection{1. "Contextes" et "conditions précises d'utilisation"}

Dans des grammaires dont la perspective est manifestement linguistique, telles que celle de Larreya et Rivière (1999a, pp. 46, 51, 52), on trouve les remarques suivantes :

la forme BE + ING est d'un emploi difficile quand le sujet n'est pas agent, c'est-à-dire avec les états. Elle n'est pourtant pas impossible (...); les états sont difficilement compatibles avec la forme BE + ING, même dans une situation particulière (...); en ce qui concerne les verbes exprimant un état, il faut se méfier. Voici des listes. Elles ne peuvent pas être complètes mais elles contiennent la plupart des verbes d'état courants non compatibles avec la forme BE + ING dans la plupart des contextes.

7 Les états constituent d'ailleurs pour ces auteurs les seuls "obstacles à l'emploi de la forme BE + ING" (p. 51 sq.). Cette approche est toutefois précisée dans Larreya (1999b, p. 145) :

un autre cas particulier est celui des verbes dits "non progressifs" ('know', 'see', 'understand', etc.) On sait qu'il est inexact de dire que ces verbes sont incompatibles avec BE + ING, mais il n'en reste pas moins que, dans la plupart des contextes, ils ne peuvent être utilisés qu'à la forme simple. Cette incompatibilité relative s'explique sans difficultés, du moins pour ce qui est de son principe général, dans le cadre des analyses de BE + ING de type aspectuel : même s'ils se situent dans le temps et peuvent avoir une durée, les états de type 'know the answer' ou 'weigh 10 tons' constituent un tout homogène, et le découpage de ce tout en "instants" qui se succèdent ne peut pas avoir grand sens. (...) quelles sont les conditions précises dans lesquelles un verbe "non progressif" peut (ou ne peut pas) être utilisé avec BE + ING ?

\subsubsection{La recatégorisation}

8 L'explication par la récatégorisation du "verbe d'état" en "verbe d'action" est proposée par Bouscaren et Persec (1998, pp. 107-108), par exemple à propos de "I’m loving this" : 
$\underline{\text { love }},(.$.$) est généralement classé parmi les procès statifs, lesquels sont d'ordinaire$ réfractaires à la forme BE -ING. (...) Dans le cas d'un verbe d'état comme love, l'emploi de cette forme a pour effet de le recatégoriser en verbe d'action.

9 Cette approche suppose la validité, qui ne va pas de soi, de l'interprétation conventionnelle qui associe l'emploi de BE + -ING à une "action". De plus, Bouscaren et Persec décrivent la recatégorisation du verbe seul (ici : "love"), sans envisager ce qui se produit sur le sujet, dont on peut pourtant poser qu'il fait partie du procès ( $c f$. note 3 et 3.1. et 3.2).

\subsection{3. Énoncés métalinguistiques}

Rappelons aussi l'apport décisif de la grammaire méta-opérationnelle, qui met en valeur, par différentes approches, la nature "métalinguistique" des énoncés en BE + -ING. Adamczewski rejette la valeur aspectuelle "progressive" de BE + -ING et conclut (1982, p. 57) que, dans la forme BE + -ING, le marqueur -ING a pour rôle de

signaler le dépassement du stade purement verbal du prédicat et, conséquence normale, la disparition du sujet grammatical.

11 Adamczewski n'en dit pas davantage sur la nature de ce "dépassement", mais pour ce qui concerne le sujet, nous pourrons faire le rapprochement avec les analyses de Gauthier (1991, p. 87) qui relève la perte fréquente du terme de départ dans les constructions prépositionnelles en -ING (cf. 2.2.1).

Delmas (1993, p. 126) souligne quant à lui la "massification du prédicat" due à la présence de -ING. Une relation $\mathrm{aRb}$, associée à $\mathrm{BE}+-\mathrm{ING}$, pourrait se présenter schématiquement ainsi : a // $\mathrm{Rb}$, ce qui suggère également que la relation prime sur les éléments qui la constituent.

Enfin, Lapaire et Rotgé (1991, p.413), indiquent que dans un énoncé en BE + -ING, le prédicat est transformé

en caractérisation (descriptive, qualitative, appréciative ou autre) du sujet grammatical (...) ; le parallèle entre "my hands are cold" et "my hands are shaking" est saisissant: dans un cas comme dans l'autre, ce qui vient à droite de $\mathrm{BE}$ est présenté comme un état du sujet grammatical.

\subsection{Hypothèse}

Face au constat selon lequel certains verbes s'emploient difficilement avec BE + -ING plusieurs approches semblent possibles : faire des listes d'exceptions ; faire apparaître que dans tous les énoncés en BE + -ING, les procès relèvent du type processus (Furmaniak, 2005), ou au contraire faire apparaître la nature stative des procès en BE + -ING, justifiant ainsi la moindre fréquence de certains procès avec BE + -ING. C'est cette dernière hypothèse que nous privilégions.

Pour cela, nous reprenons à notre compte la nature métalinguistique ${ }^{4}$ des énoncés en $\mathrm{BE}+$ -ING, dont la conséquence est que le procès y a le statut d'objet de discours. Notre hypothèse est que, dans un tel statut, les distinctions état / processus et agent / siège ne sont pas pertinentes car elles relèvent d'un niveau linguistique (la sémantique), et non pas métalinguistique. L'anaphorisation associée à BE + -ING (Adamczewski, 1982, pp. 58 sq. , Girard, 2000, pp.129-138) ferait passer à l'arrière-plan les propriétés primitives des notions, au profit d'une stabilisation du procès, objet de discours. Il devient alors possible 
de rendre compte du fait que les lexèmes prototypiquement associés à un état dans l'Aktionsart se trouvent moins fréquemment employés avec BE + -ING.

Nous rassemblons ci-dessous des éléments qui pourraient permettre de valider cette hypothèse.

\section{Vers une uniformisation de la nature du procès dans les énoncés en BE + -ING}

\subsection{Sortir d'une approche lexicale figée}

Notre hypothèse favorise une valeur uniforme du procès dans les énoncés en BE + -ING, celle d'un état. Elle suppose donc de sortir d'une approche lexicale figée pour adopter une approche de type notionnel liée aux opérations énonciatives. L'hypothèse proposée s'appuie en cela sur le fait qu'un même lexème puisse désigner plusieurs notions ${ }^{5}$, selon les opérations énonciatives effectuées.

\subsubsection{Le cas des verbes}

Relevant du mode énonciatif du commentaire, l'énoncé en BE + -ING engage un point de vue de l'énonciateur, point de vue de nature souvent aspectuelle; l'énoncé est donc modalisé, au sens large du terme, ce qui permet notamment à l'énonciateur de jouer sur la valeur référentielle du prédicat. Cela est en effet possible puisque dans la langue, les notions différentes sous un même lexème ne sont pas rares. C'est fréquemment le cas de / have/ et /be/ par exemple, qui figurent des notions différentes selon qu'on a "have a house" ou "have a walk", "be mad" ou "be a fool". Pour Bjerre (1999) et Harley (2003), cela est lié à leur capacité à être des verbes-supports ("light verbs"). Toutefois, d'autres lexèmes ne présentant pas cette capacité peuvent figurer des notions différentes selon le contexte et la situation repère ; on peut observer par exemple /think/ et /hope/ dans les énoncés (1) à (3) :

Perhaps in the vegetarian future people will use adultery as an alibi for eating meat (...) [in] seedy beef hotels that rent private dining rooms by the hour. How come I'm thinking about beef? I was thinking about ... about William James and consciousness as a stream. (T, p. 3)

(1a) Perhaps in the vegetarian future people will use adultery as an alibi for eating meat (...) [in] seedy beef hotels that rent private dining rooms by the hour.?How come I think about beef? I thought about ... about William James and consciousness as a stream.

(2) (...) reporting what people say to each other but never telling the reader what the characters are thinking. (T, p. 63)

(2a)?(...) reporting what people say to each other but never telling the reader what the characters think.

(3) I was not a little dismayed to discover on my arrival that Russell Marsden had already departed to the South of France, as I was hoping to get some tips from him about how to run the course. (T, p. 12)

(3a)?I was not a little dismayed to discover on my arrival that Russell Marsden had already departed to the South of France, as I hoped to get some tips from him about how to run the course.

Si on supprime BE + -ING (énoncés (1a)-(3a)), la compatibilité avec les contextes devient discutable : en (1a), la présence de "how come", qui marque une investigation des pensées 
préalables du narrateur, n'est guère compatible avec l'aspect $\varnothing$ du présent simple ; en (2a), la forme -ING de "reporting" et de "telling what" ne s'accommode pas de l'aspect $\varnothing$ du présent simple; enfin, le contexte de (3), notamment avec "on my arrival" et "as" implique la présence d'un commentaire, rendu éventuellement par "as I had hoped", mais non par "I hoped". Ces quelques remarques suggèrent que les notions /think/ et /hope/ ne sont pas les mêmes dans les deux séries d'énoncés.

La modification notionnelle n'est ainsi pas réservée aux verbes-supports; elle n'est pas non plus due à la présence de BE + -ING puisque par exemple, "swam", dans "John swam" est signalé par Dowty $(1979$, p. 61) comme pouvant avoir deux interprétations relevant de deux notions différentes: si on sait que John parcourt régulièrement à la nage une distance fixe, il s'agit d'une notion à valeur télique, paraphrasable par (4) :

(4) John swam the distance he usually does.

Dans le cas où, au contraire, il n'y a pas de connaissance partagée concernant une distance habituellement parcourue par John, la notion se distingue de la première par sa valeur atélique. Les opérations énonciatives mises en œuvre sont un lieu de liberté pour l'énonciateur, liberté exemplifiée par Bouscaren et al. (1993, p. 21) avec BE +-ING et les procès téliques :

des procès téliques peuvent recevoir une interprétation purement qualitative où le terme n'est pas pris en compte. Ici encore c'est la mise en œuvre des opérations énonciatives qui permettra une interprétation qualitative non quantitative :

He was slowly recovering from his illness

$\mathrm{He}$ is repairing the car.

L'opération énonciative marquée par BE + -ING filtre la valeur télique notionnelle : elle affecte ainsi la notion pour en sélectionner certaines caractéristiques.

De même, on trouve des exemples avec des procès habituellement considérés comme statifs, donc a priori non bornés, qui, par opération énonciative marquée par HAVE -EN, se trouvent affectés d'une borne de droite; c'est le cas de /be happy/ dans l'exemple (5):

(5) She'd been happy until she met him.

À l'inverse, on trouve des procès habituellement considérés comme processus qui, dans les constructions en HAVE d'expérience, reçoivent une interprétation stative, ainsi que le montre Harley (1998, p. 195) :

experiencer readings [of HAVE] with apparently eventive complements can in fact be seen to necessarily have stative interpretations.

C'est par exemple le cas de /steal/ dans l'énoncé (6) :

(6) I had my bike stolen yesterday.

Selon Ritter et Rosen (1997, p. 318), il en est de même avec HAVE auxiliaire, qui peut permettre de construire une interprétation stative de /read/ (énoncés 7-8) :

auxiliary have provides an additional association between the event or state and the (derived) subject by making the event/state a property of the subject.

Ritter et Rosen appuient leur raisonnement sur les énoncés (7) et (8) :

(7a) John read the New York Times twice.

(7b) John has read the New York Times twice.

(8a) John read a book twice.

(8b) John has read a book twice.

En (7a) et (8a), l'objet réfère à un exemplaire unique du New York Times ou de livre, lu deux fois par John. En $(7 b)$ et ( $8 b)$, l'objet peut référer à deux exemplaires distincts; dans cette interprétation, les énoncés dénotent une propriété de John, paraphrasable par "John is a two-time New York Times/book reader". Le sujet est siège d'une propriété : la 
notion verbale /read/ n'est pas seule à être affectée, le sujet l'est également. Cet effet de HAVE auxiliaire sur le sujet n'est pas sans rappeler la "relation statique" établie par HAVE et décrite par Bouscaren et al. (1982, p. 58) dans des énoncés du type "this man has blue eyes".

$\mathrm{BE}+$ - ING pourrait avoir un fonctionnement similaire, et affecter la nature du procès ( $c f$. note 3) jusqu'à la modifier.

\subsubsection{Le cas des noms}

De façon cohérente, nous observons un parallèle entre le groupe verbal et le groupe nominal, pour ce qui concerne la notion. En effet, l'examen fait par Gauthier (1986) de quelques emplois annoncés comme "marginaux" de l'indéfini A va dans le même sens. Ces emplois sont dits "marginaux" parce que l'indéfini $\mathrm{A}$ y est associé à des termes qui renvoient habituellement à du continu : "a disgrace", "a shame", "a relief", ou bien à des expressions non pluralisables : "he just loves a mess" ; "have a go" ; "have a good think". Gauthier remarque (op.cit., p. 197) que certains de ces termes se situent à la limite du verbal et du nominal. Or s'ils sont associés à une situation repère bien particularisée, il y a discrétisation. Gauthier (1999, pp. 86-87) revient sur ces emplois, pour poser que :

la découpe dont a est la trace ne passe pas par la référence explicite à la classe (...)

On passe ainsi d'une logique du tout ou rien à une logique du plus ou moins.

La discrétisation, qui n'implique pas la cardinalité, se positionne comme une souscatégorie du discontinu (Gauthier, 1986, p. 195); elle permet toutefois la réitération dans une situation identique d'un "quantum itérable mais non cumulable" (op. cit., p. 196) comme dans "another go". Il y a donc tout lieu de penser qu'une modification s'effectue par opération énonciative et concerne les propriétés physico-culturelles de la notion.

Gauthier remarque d'ailleurs que ces termes sont fréquemment situés dans des énoncés fortement modalisés, tels que "What a disgrace !" ou "What a pity !", où l'"application d'une modalité de type 3" par l'énonciateur peut être glosée par "il est regrettable, dommage que" ( op.cit., p. 196). Le fonctionnement similaire de EVERY est analysé par Chuquet (1997, p.130) sur les expressions du type "every assistance", "every evidence", "every encouragement".

Ces quelques remarques autour du groupe nominal illustrent la latitude dont l'énonciateur dispose dans le traitement de la notion.

\subsection{Effet des morphèmes constitutifs de BE + -ING}

\subsection{1. -ING}

Le morphème -ING a fait l'objet de nombreuses publications et nous relevons ci-dessous quelques éléments utiles à notre réflexion dans la mesure où ils suggèrent des modifications du prédicat.

Dans l'histoire de la langue anglaise, -ING semble avoir une double origine : le participe présent, dont la marque -ENDE devient -ING en Moyen Anglais, et le gérondif, forme nominale du verbe, longtemps employée en contexte prépositionnel après BE : BE ON / IN / UPPON (sic) (Elsness, 1994, pp. 7, 13). L'emploi prépositionnel implique que la relation soit validée préalablement à la localisation par la préposition. Cette validation préalable à l'énoncé en BE +-ING pourrait être à la source de la plupart des remarques qui suivent. 

c'est-à-dire :

à un stade antérieur à la réalisation lexicale sous forme nominale ou verbale : on a ainsi la notion prédicative /read/ qui n'est ni un nom ni un verbe. Or la forme lexicalisée reading possède justement des propriétés nominales et des propriétés verbales; dans certains de ses emplois, elle garde toutes les propriétés du verbe (ex : I like reading a book) ou au contraire elle peut les perdre toutes pour prendre celles d'un nom (ex : I don't like John's reading of this poem). (...) on considérera donc que ce terme [reading] renvoie à un ensemble d'occurrences de la notion / $\mathrm{read} /$, autrement dit à un domaine notionnel constitué d'occurrences qualitativement identiques.

Dans cette logique, la nature du procès ne devrait pas être un critère pertinent, ce qui permettrait alors à l'ensemble des notions d'être compatibles avec BE + -ING. C'est d'ailleurs l'observation des occurrences de BE + -ING avec les verbes d'état mental qui conduit Souesme (2000, p. 140) à souligner que l'emploi de BE + -ING est indépendant du type de procès.

Groussier (2002, p. 202) mentionne également la notion, évoquant en conclusion une " modification notionnelle", sans vraiment la détailler :

l'interprétation par l'énonciateur de l'occurrence selon son propre point de vue peut aller jusqu'à une modification notionnelle; avec la forme BE + ING on ne travaille plus sur la notion de procès mais sur la notion d'état de participation, soit de l'actant principal soit de l'actant secondaire.

Par ailleurs, l'hypothèse de la nominalisation par -ING, surtout développée par Adamczewski (1982, pp. 56-58) et Cotte (1997, p. 98), va dans le sens de la perte de son statut verbal par le prédicat. Pour Cotte, "birds were whistling" s'appuie sur la nominalisation de "birds whistled", ce qui repousse le prédicat /whistle/ à l'arrière-plan. Gauthier, quant à lui, indique explicitement qu'il y a "déverbalisation" (1991, p. 82).

L'absence de diathèse associée à $B E$ + -ING jusqu'au début du dix-huitième siècle (Jespersen, 1931, p. 211), avec des énoncés comme "The house is building", va également dans le sens du statut nominal du terme affecté de -ING.

Des arguments supplémentaires en faveur de la neutralisation des caractéristiques des éléments constitutifs du procès figurent dans les travaux de Gauthier, notamment la perte du rôle agentif du sujet dans les constructions prépositionnelles en -ING (Gauthier, 1991, pp. 90-91): par exemple, dans "the coming of the British" ("the $x_{1}$ of $x_{2} "$ "), la thématisation du prédicat manifeste que ce ne sont pas les Britanniques mais leur arrivée qui intéresse l'énonciateur. Ainsi, "the $x_{1}$ " situe le prédicat au premier plan avec une opération de fléchage qui reprend à l'identique un préconstruit dans lequel on peut penser que le terme de départ n'était déjà plus agent : on peut supposer en effet que du fait que le fléchage marque l'identité entre le référent et le préconstruit, le rôle agentif du sujet dans le préconstruit est réduit voire nul. Le procès tout entier (cf. note 3 ) serait ainsi affecté par les opérations énonciatives.

Gauthier (1991, p. 87) montre de plus que dans ces expressions, le terme de départ peut être perdu, même s'il est le plus souvent récupérable grâce au contexte, comme c'est le cas par exemple de "the finding of the body" ou de "the bringing up of children". Pour Gauthier, cela indique que c'est la relation entre les éléments qui prime aux yeux de l'énonciateur, au détriment des éléments mis en relation:

la construction prépositionnelle ne semble pas renvoyer à un schéma <arb> mais plutôt à une cohésion particulière posée entre le procès et son objet. 
Il n'y a donc pas vraiment de pertinence à poser une distinction entre verbes d'état et verbes de processus sous l'angle de la compatibilité avec -ING, hormis pour chercher à comprendre pourquoi les verbes d'état sont plus réticents à être nominalisés (cf. 3.2.).

\subsection{2. $\mathrm{BE}$}

Dans la théorie des opérations énonciatives, BE est associé à la valeur "égal" de l'opérateur de repérage. Les termes concernés, de part et d'autre de $\mathrm{BE}$, sont le sujet de l'énoncé (S) et le prédicat $(\mathrm{P})$. BE se trouve donc au centre d'un équilibre et la présence de $\mathrm{BE}$ dans les énoncés en $\mathrm{BE}+$ - ING en fait fondamentalement des énoncés binaires.

Le fait que BE exprime l'état permet de considérer que le lien S-P est stabilisé dans la situation repère; le prédicat évoque une caractéristique, posée comme équivalente au sujet dans la situation repère : le sujet devient donc le siège de cette caractérisation. Le sujet est ainsi installé dans une stabilité proche de l'état, le rôle de ce dernier étant précisément de définir le sujet.

43 Dans les exemples (9) à (11), on relève la présence d'éléments (repérés par l'italique) qui indiquent en effet que le sujet est totalement associé au procès, défini par lui, ce qui exclut tout autre procès dans la situation repère :

(9) Don't disturb me, I'm listening to some music!

(10) I decide not to disturb her; utterly absorbed in what she's reading, she' s seen the Rockies before. (VM, p. 91)

(11) They were safe, across the ocean, locked in their seasonal rhythms, consumed by their various passions. They were taking Portuguese lessons, they had written. And growing orchids. (VM, p. 177)

Pour chaque situation repère, $S$ est défini par la propriété /be listener/ (9), /be reader/ (10), /be student of Portuguese/ ou /be orchid grower/ (11). Chacun de ces états étant exclusif ${ }^{6}$, il est la cause ou la justification d'un comportement ou d'un jugement, pouvant être paraphrasés ainsi :

(9a) Don't disturb me because / as I am reading.

(10a) I decide not to disturb her because / as she is reading.

(11a) They were safe because / as they were locked in their seasonal rhythms.

On observe pourtant dans les énoncés (9) à (11) l'absence de connecteur entre les propositions. C'est $\mathrm{BE}+$ - ING lui-même qui véhicule la valeur de propriété exclusive associée au sujet dans la situation repère. D'ailleurs, lorsqu'un connecteur est présent, il peut exprimer simplement la coordination entre les propositions, même si la relation effective a une nature explicative comme en (12), dont la glose pourrait être : Gwenneth n'a pas d'argent puisqu'elle consacre tout son temps à la préparation de sa thèse :

(12) Gwenneth was working on a Ph.D. and, for the most part, was without of money. (VM, p. 144)

Des éléments contextuels tels que ceux relevés dans les énoncés (9) à (11) ne sont pas toujours explicites. Ainsi, dans l'exemple (13), c'est à la fois le repérage temporel et la complémentation verbale qui jouent un rôle essentiel pour exprimer la stabilité proche de l'état :

(13) How come I'm thinking about beef? I was thinking about ... about William James and consciousness as a stream (T, p. 3)

Dans cet énoncé, le marquage temporel associé à deux situations repères distinctes ("I was thinking" puis "I’m thinking") appuie l'interprétation proposée pour les exemples (9) 
à (11) : en effet, le changement de complément associé à /think/ montre bien que le sujet "I" est totalement caractérisé par "think about William James", puis par "think about beef". Le comique de l'énoncé provient de l'association séquentielle mais totale du sujet de l'énoncé à des pensées de nature bien variable ... On comprend alors mieux la difficulté qu'il y a à produire (1a)-(3a) avec le même contexte que celui des énoncés (1)-(3) (cf. 2.1.1).

Dans d'autres contextes, on relève que la valeur stative de la relation S-P est corroborée par la présence d'éléments statifs (repérés par l'italique) qui expriment des états de $\mathrm{S}$ :

(14) Both Robert and Lila, each enclosed in a separate vision, could imagine houses filled with lighted rooms, and these rooms - like the one they were sitting in were softened by the presence of furniture, curtains, carpets, men and women and children, and by that human contrivance that binds them together. (VM, p. 183)

(15) In the seat ahead of us is a young man wearing a bright blue jacket - I remember that once I had a similar jacket in a similar hue. (...) From where I am sitting I can see his intense, eager trigger hand. (...) I tell myself that I would give a great deal to be in that young man's shoes. (VM, pp. 91-92)

La même valeur de stabilité dans la situation repère est encore visible dans les énoncés qui présentent un commentaire de nature explicitement métalinguistique ( $c f$. note 4) comme (16) :

(16) 'Look,' he said to Bernie, (...), historians in the past thought history as a continuum. And we haven't been able to see what was patently obvious. (...) It's our curse to overlook beginnings. Beginnings just don't register because we're locked in our vision of the status quo. We can't even be bothered to acknowledge the feeble stirrings - '

'You're not suggesting,' Bernie said, 'that no one acknowledged the storming of the Bastille?'

'Let's just say they didn't know what it signified.' (HHS, p. 5)

Les occurrences de ce type, avec/suggest/, mais aussi /say/, /kid/, /joke/, /tell/, etc. sont fréquentes. La stabilité en jeu concerne l'adéquation entre l'énoncé repère et son interprétation. Dans l'exemple (16), la présence de "let's just say" confirme cette valeur.

Si le débat sur la durée de validité des procès en BE +-ING est clos (Rivière, 1993), il reste à souligner qu'un état peut fort bien être transitoire, comme le montrent les contextes cidessus, et qu'il ne devrait pas y avoir incohérence entre BE + -ING et la construction d'un état par opération énonciative.

\section{Conséquences de notre hypothèse}

\subsection{BE + -ING et le franchissement de borne}

La proposition selon laquelle, dans les énoncés en BE + -ING, le sujet soit siège d'une propriété, et non pas agent, amène deux réflexions: premièrement, cette proposition reste-t-elle compatible avec le raisonnement convaincant de Souesme (1992, p. 88 sq.) qui montre que $\mathrm{BE}+-\mathrm{ING}$ est associé au franchissement de la borne de l'extérieur (E) vers l'intérieur (I) du domaine notionnel? Deuxièmement, ces franchissements de borne devraient supposer l'agentivité du sujet ; mais de quel sujet s'agit-il ?

Prenons le cas des verbes avec aspect lexical, tel que /begin/, /start/, /cease/, /finish/, / stop/. Ces verbes signalent un moment du procès. Or, parmi ces verbes, seuls /begin/ et / cease/ sont habituellement réputés compatibles avec des procès de type état (repérés par l'italique) :

(17) I'm beginning to see what you mean. 
(18) She ceased to be a farmer when GMOs appeared. que peu de notions associées à des propriétés physico-culturelles de type état avec $\mathrm{BE}+$ ING car il se produit alors une redondance. L'évolution de la langue, avec la restriction graduelle des emplois de $\mathrm{BE}+-\mathrm{ING}$, souligne la tendance à éliminer ces redondances : ainsi /flow/ ou /run/ étaient employés avec BE + -ING dans des contextes où l'anglais contemporain utilise les temps simples. Elsness (1994, p. 7) cite deux exemples en Vieil Anglais: "from Danai that river which is running from northern-part" et "that this river which is flowing all Egyptians' land". De même, certains emplois de BE + -ING en Vieil Anglais peuvent être ramenés à des formes adjectivales dont la valeur est l'expression d'un état: "Therefore that which is in its Nature differing from the chief Good, cannot be said to be the Good itself", dans lequel "is differing" est très proche de "is different" (Elsness, 1994, p. 20).

Lorsque la redondance est choisie par l'énonciateur, c'est essentiellement pour souligner que la relation est associée à une situation repère particularisante. Cela permet à l'énonciateur de "qualifier ou de quantifier le noud prédicationnel" (Adamczewski, 1982, p. 75) et de construire une interprétation différente de celle produite sans BE + -ING ((24a)-(29a)) ; cela concerne des notions aussi variées que /plan/, /think/, /have/, /feel/, ou encore /lie/ dans les énoncés (24) à (28) : 
(24) "It just kills me to leave this place," the young woman says. She's following us through the rooms, pointing with unmistakable sorrow at the wall where they were planning to put up shelving, at the hardwood floors they were thinking of sanding. (VM, p. 98)

(24a) "It just kills me to leave this place," the young woman says. She's following us through the rooms, pointing with unmistakable sorrow at the wall where they planned to put up shelving, at the hardwood floors they thought of sanding.

Because of the way she had said this, and the way she had squeezed her eyes shut, Hélène knew her mother was thinking about Roger, the man in Winnipeg she was in love with. (VM, p. 41)

(25a) Because of the way she had said this, and the way she had squeezed her eyes shut, Hélène knew her mother thought about Roger, the man in Winnipeg she was in love with.

(26) I was thinking to myself, how agreeable that is, what a nice time I'm having, but there's one thing missing -someone to share it with, or report it to. (T, p. 65)

(26a) I thought to myself, how agreeable that is, what a nice time I have, but there's one thing missing -someone to share it with, or report it to.

(27) You could buy a happiness-in-your-new-home card, (...) even a spectacular three dollar pop-up card announcing to the world that you were feeling underappreciated. (VM, p. 66)

(27a) You could buy a happiness-in-your-new-home card, (...) even a spectacular three dollar pop-up card announcing to the world that you felt under-appreciated.

(28) He sat down on the bed and stared around him. Nearly everything in here was broken. The month-old cine-cinema was lying on top of a small, working tank. (HPPS, p. 32)

(28a) He sat down on the bed and stared around him. Nearly everything in here was broken. The month-old cine-cinema lay on top of a small, working tank.

Notre hypothèse permet ainsi de poser qu'il n'y a pas, a priori, de verbes réfractaires à l'emploi de BE + -ING puisque ce n'est pas sur le verbe ni sur le sujet que porte la quantification ou la qualification mais sur leur relation.

\section{Conclusion}

A plusieurs reprises, nous avons évoqué la notion, sous l'angle des modifications qui pourraient l'affecter. L'hypothèse de la neutralisation des caractéristiques notionnelles a été proposée. $\mathrm{BE}+$ - ING pourrait marquer une prise en charge énonciative forte par laquelle l'énonciateur explicite la mise en relation prédicative, en la quantifiant ou en la qualifiant. Cette mise au premier plan du rôle de l'énonciateur donnerait non seulement au sujet, mais aussi au prédicat le statut d'objets de discours, neutralisant de ce fait les traits sémantiques qui leur sont associés dans l'Aktionsart ou notionnellement.

61 Nous pouvons alors rapprocher BE + -ING de l'aspect en creux marqué par $\emptyset$ et considérer leur fonctionnement en système. $\varnothing$ et $\mathrm{BE}+$ - ING sont habituellement traités sous l'angle du rôle de l'énonciateur: pour reprendre les termes d'Adamczewski, il y a simple connexion sémique avec $\varnothing$ (rôle minimal de l'énonciateur) et énoncé métalinguistique avec BE + -ING (rôle maximal de l'énonciateur). Si on aborde maintenant ces marqueurs sous l'angle du procès, $\varnothing$, par effacement de l'énonciateur, laisse transparaître la nature $\mathrm{du}$ procès alors que $\mathrm{BE}+-\mathrm{ING}$ semble mettre au premier plan de l'énoncé sa prise en charge par l'énonciateur. Cela implique la "désagentivisation" du sujet au profit du statut de siège (Adamczewski, 1992, p. 76) et affecte la nature du prédicat, par intrusion énonciative ayant pour effet de neutraliser les types de procès : le procès tout entier est affecté d'une valeur d'état, le temps d'un énoncé. 


\section{BIBLIOGRAPHIE}

ADAMCZEWSKI, H. (1982, rééd. 1997). Grammaire linguistique de l'anglais. Paris, Armand Colin.

BJERRE, T. (1999). "Event Structure and Support Verb Constructions". In Todirascu, A. (ed.), Proceedings of the ESSLLI Student Session. p. 3 sq.

BOUSCAREN, J., CHUQUET, J. et FILHOL-DUCHET, B. (1982). "HAVE opérateur de localisation". Cahiers de Recherche en Grammaire Anglaise. t. 1, pp. 55-72.

BOUSCAREN, J. et DESCHAMPS, A. (1991). "Réexamen de la typologie des verbes anglais". Cahiers Charles $V . \mathrm{n}^{\circ} 13$ (Travaux de linguistique énonciative), Paris, Institut d'Anglais et Université Paris VII, pp. 7-23.

BOUSCAREN, J., DESCHAMPS, A. et MAZODIER, C. (1993). "Eléments pour une typologie des procès". Cahiers de Recherche en Grammaire Anglaise. t. 6 (Types de procès et repères temporels), Gap et Paris, Ophrys, pp. 5-34.

BOUSCAREN, J. et PERSEC, S. (1998). Analyse grammaticale dans les textes. Gap et Paris, Ophrys.

CHUQUET, J. (1997). "Each, every et la délimitation des occurrences". Cahiers de Recherche en Grammaire Anglaise. t. 7 (La composante qualitative : déterminants et anaphoriques), Gap et Paris, Ophrys, pp. 127-144.

COTTE, P. (1997). Grammaire linguistique. Paris, Didier-Erudition.

DELMAS, C. (1993). "Le réinvestissement modal de certaines formes verbales". Opérations énonciatives et interprétation de l'énoncé. Danon-Boileau, L. et Duchet, J.-L. (eds), Gap et Paris, pp. 115-133.

DOWTY, D.R. (1979). Word Meaning and Montague Grammar. Dordrecht, Reidel.

ELSNESS, J. (1994). "On the progression of the progressive in early Modern English". ICAME Journal. $\mathrm{n}^{\circ} 18$, pp. 5-25.

FURMANIAK, G. (2005). "The BE + -ING form: Progressive aspect and metonymy". CORELA. vol. 3, n - 2, http://www.revue-corela.org (consulté en janvier 2006).

FURULI, R. (1997). "Aktionsart vs Aspect". http://www.ibiblio.org/bgreek/test-archives/ html4/1997-05/18805.html, consulté en mars 2006.

GAUTHIER, A. (1986). "A propos de quelques emplois marginaux de l'indéfini $a$ ". Cahiers Charles $V$. $\mathrm{n}^{\circ} 8$ (Lignes de partage), Groussier, M.-L. (éd.), Paris, Institut d'Anglais et Université Paris VII, pp. 193-204.

GAUTHIER, A. (1991). "Constructions en -ING. (The) Teaching (of) English in France". Cahiers Charles V. $\mathrm{n}^{\circ} 13$ (Travaux de linguistique énonciative), Groussier, M.-L. (éd.), Paris, Institut d'Anglais et Université Paris VII, pp. 79-101.

GAUTHIER, A. (1999). "Singuliers non pluralisables et construction de l'unité". CYCNOS. vol. 16, n • 2, Souesme, J.-C. (éd.), Nice, Université de Nice - Sophia-Antipolis, pp. 83-97.

GIRARD, G. (2000). "Be + V-ing, rôle anaphorique ?". CYCNOS. vol. 17, nº spécial, Souesme, J.-C. (éd.), Nice, Université de Nice - Sophia-Antipolis, pp. 129-138. 
GROUSSIER, M.-L. (2000). "Be + ing ou la prédication de l'état de partcipation de l'actant principal (ou secondaire) à une occurrence de procès". CYCNOS. vol. 17, n spécial, Souesme, J.-C. (éd.), Nice, Université de Nice - Sophia-Antipolis, pp. 189-202.

HARLEY, H. (2003) "How do verbs get their names? Denominal verbs, Manner Incorporation and the ontology of verb roots in English". http://w3.arizona.edu/ ling/hh (consulté en mars 2006).

HARLEY, H. (1998). "You're having me on!: aspects of have". In : Guéron, J. et Zribi-Hertz, A. (eds), La grammaire de la possession. Nanterre, Paris X, pp. 195-226.

JESPERSEN, O. (1931). A Modern English Grammar on Historical Principles. t. 4, Copenhague, Ejnar Munksgaard.

LAPAIRE, J.-R. et ROTGE, W. (1991). Linguistique et grammaire de l'anglais. Toulouse, Presses Universitaires du Mirail.

LARREYA, P. et RIVIERE, C. (1999a). Grammaire explicative de l'anglais. Harlow, Addison Wesley Longman Limited.

LARREYA, P. (1999b). "BE +-ING est-il un marqueur d'aspect ?". ANGLOPHONIA. vol. 6, Toulouse, Presses Universitaires du Mirail, pp. 135-150.

RITTER, E. et ROSEN, S. (1997). "The function of have". Lingua. n 101, pp. 295-321.

RIVIERE, C. (1993). "Illusions de la durée". Cahiers de Recherche en Grammaire Anglaise. t. 6 (Types de procès et repères temporels), Gap et Paris, Ophrys, pp. 113-117.

SOUESME, J.-C. (1992). Grammaire anglaise en contexte. Gap et Paris, Ophrys.

SOUESME, J-C. (2000). "Le perfect en be + ing et la construction d'une contiguité". CYCNOS. vol. 17, $\mathrm{n}^{\circ}$ spécial, Souesme, J.-C. (éd.), Nice, Université de Nice - Sophia-Antipolis, pp. 139-158.

Corpus extrait de :

David LODGE, Thinks..., Londres, Penguin Books, 2001 (T)

Joanne K. ROWLING, Harry Potter and the Philosopher's Stone, Bloomsbury, Londres, 1997 (HPPS)

Carol SHIELDS, Various Miracles, Penguin Books, Londres, 1985 (VM)

Carol SHIELDS, Happenstance: the Husband's Story, Flamingo, Londres, 1994 (HHS)

\section{NOTES}

1. Que soit ici vivement remercié Philippe Rothstein (Université Paul Valéry, Montpellier 3) pour sa lecture très attentive d'une version antérieure et pour les pistes de réflexion qu'il a suggérées et qui ont permis d'enrichir cet article. Notre reconnaissance va également aux relecteurs d'une précédente version, dont les remarques ont permis d'affiner ce travail.

2. C'est nous qui soulignons. Cette remarque s'applique à tous les éléments soulignés dans les citations et les exemples, pour des raisons autres que métalinguistiques. Par ailleurs, les verbes en BE+-ING faisant l'objet d'une analyse apparaissent en caractères gras dans les exemples.

3. Le terme "type de procès" réfère manifestement ici à la distinction entre "verbe statif" et verbe non statif; "procès" nous semble être employé ici au sens de "verbe". Pour notre part, dans la suite de l'article, nous appliquons le terme de "procès" à la réalité sémantique issue de la mise en relation du prédicat et des arguments. 
4. Nous qualifions de "métalinguistique" la valeur issue de l'emploi de marqueurs destinés à commenter les choix linguistiques de l'énonciateur. L'énoncé (ou une partie de l'énoncé) est dans ce cas objet de discours pour l'énonciateur.

5. Nous évoquons ici la distinction entre une forme lexicale unique (par exemple, "time") et les notions qu'elle désigne : /fois/ dans "four times", /temps + approche quantifiable ou quantifiée/ dans "much time", /temps + approche qualitative/ dans "time is money". Cette distiction n'est pas incompatible avec l'existence d'un invariant puisque pour l'exemple pris, c'est la caractérisation temporelle qui constitue l'invarariant.

6. Dans l'exemple (11), même si deux propriétés sont évoquées successivement ("they were taking (...). And growing orchids."), cela n'est pas incompatible avec une interprétation exclusive puisque la seconde propriété est présentée comme un ajout de second plan : on remarque en effet un point, puis la coordination par "And" et l'absence de "they were".

7. La définition de l'aspect donnée par Furuli (cf. 1.1.) ("the way that language user decides to portray that action") reste valable si on interprète "portray that action" comme l'image de la mise en relation du sujet et du prédicat par l'énonciateur.

\section{RÉSUMÉS}

$\mathrm{BE}+$ - ING est-il ou non compatible avec des procès de type état? Dans la littérature, cette question donne lieu à des réponses variables, souvent accompagnées de listes d'exceptions. Dans cet article, nous formulons l'hypothèse selon laquelle, dans tout énoncé en BE + -ING, le point de vue aspectuel inséré par l'énonciateur agit comme un filtre des propriétés prototypiquement associées à la notion, installant temporairement le procès, dans une stabilité de type état; le sujet est alors siège d'une propriété véhiculée par le prédicat affecté de -ING. Employé avec des procès de type état, $\mathrm{BE}+-\mathrm{ING}$ crée une redondance, choisie par l'énonciateur si et seulement si celui-ci la juge nécessaire dans le contexte.

Is $\mathrm{BE}+-\mathrm{ING}$ compatible or not with stative processes? In the literature, various answers are given to the question, and they usually appear with lists of exceptions. This article is about a hypothesis according to which, in any BE + -ING utterance, the aspectual point of view filters the prototypical properties associated with the notion, temporarily giving the process a stative value; the subject becomes thus the seat of the property expressed by the predicate with -ING. Used with stative processes, BE + -ING induces redundancy, and is therefore chosen by the utterer only when the context requires it.

\section{INDEX}

Mots-clés : BE +-ING, procès, état, processus, aspect

Keywords : process, state, event 
AUTEUR

LAURENCE VINCENT-DURROUX

Université Paul Valéry, Montpellier 3 